\title{
Ontological Order in Scientific Explanation
}

Park, Seungbae (2003). "Ontological Order in Scientific Explanation", Philosophical Papers 32 (2): 157-170.

\author{
Seungbae Park \\ Division of General Studies \\ Ulsan National Institute of Science and Technology \\ nature@unist.ac.kr
}

\begin{abstract}
A scientific theory is successful, according to Stanford (2000), because it is sufficiently observationally similar to its corresponding true theory. The Ptolemaic theory, for example, is successful because it is sufficiently similar to the Copernican theory at the observational level. The suggestion meets the scientific realists' request to explain the success of science without committing to the (approximate) truth of successful scientific theories. I argue that Stanford's proposal has a conceptual flaw. A conceptually sound explanation, I claim, respects the ontological order between properties. A dependent property is to be explained in terms of its underlying property, not the other way around. The applicability of this point goes well beyond the realm of the debate between scientific realists and antirealists. Any philosophers should keep the point in mind when they attempt to give an explanation of a property in their field whatever it may be.
\end{abstract}

\section{Introduction}

The present paper focuses on the debate between a scientific antirealist, Kyle Stanford (2000), and a scientific realist, Stathis Psillos (2001). I criticize both philosophers, and then draw a philosophical lesson about the nature of scientific explanation. The applicability of the lesson, however, far exceeds the realm of philosophy of science. I argue that a good explanation should meet a certain conceptual requirement, which is new to the philosophy of science literature. Any philosophers should keep the requirement in mind when they attempt to give an explanation of a property in their field whatever it may be. What exactly the requirement is will become clear at the end of the paper as a byproduct after I discuss the debate between Stanford and Psillos.

The debate between Stanford and Psillos concerns the success of science, and the concept of success plays a crucial role at the end of this paper where I criticize Stanford's position. So it is useful to be precise about it at this stage. So far as I know, Larry Laudan provides the most precise definition of 'success' in the literature:

...we would say that a theory is successful if it makes substantially correct predictions, if it leads 
to efficacious interventions in the natural order, if it passes a battery of standard tests. (1981: 23)

Accordingly, I shall assume that a theory is 'successful' so long as it has worked well, i.e., so long as it has functioned in a variety of explanatory contexts, has led to confirmed predictions and has been of broad explanatory scope. (1981:23)

In short, a successful theory has high explanatory, predictive and manipulative powers. Success, I should add, is a relation among a scientific theory, the world and us. Two conditions have to be met for a theory to be successful. First, at least some of its observational consequences must be true. Second, we should recognize them to be so. Keep in mind that the truth of some observational consequence is built into success.

Scientific realists argue that a scientific theory is successful because it is approximately true, and that its key terms refer (Putnam, 1975). The theory of DNA, for instance, is successful in curing us of diseases and in producing new plants and animals because it is at least approximately true, and because DNA is real. It would be a miracle if it is completely false, and if DNA does not exist. So we have good reason to take the realist attitude toward successful theories, viz., they are approximately true, and the theoretical entities they postulate are real. This argument for scientific realism, so-called no-miracle argument, is widely regarded as the best argument for scientific realism (Kitcher, 1993; Psillos, 1999; Arabatzis, 2001), and sparked hot and voluminous debates between scientific realists and antirealists in the literature.

\section{Stanford vs. Psillos}

Stanford makes an interesting and creative antirealist suggestion that the success of a false theory be explained by its sufficient observational similarity to its corresponding true theory:

... the success of a given false theory in a particular domain is explained by the fact that its predictions are (sufficiently) close to those made by the true theoretical account of the relevant domain. (Stanford, 2000: 275)

The Ptolemaic hypothesis, for instance, was successful because it was "able to generate sufficiently similar predictions to those of the Copernican hypothesis" (2000: 274). Stanford's story might be taken as realistic on the grounds that a mention is made of a true theory. But it is antirealistic because, according to him, a given theory whose success is explained is not true. The explanation works, as long as there is a true theory that is sufficiently observationally similar to the false theory:

Notice that the actual content of the Copernican hypothesis plays no role whatsoever in the 
explanation we get of the success of the Ptolemaic system: what matters is simply that there $i s$ some true theoretical account of the domain in question and that the predictions of the Ptolemaic system are sufficiently close to the predictions made by that true theoretical account. (2002: 274)

We need to be clear about the explanans and the explanandum in Stanford's explanatory story. The explanans is observational similarity ${ }^{1}$, which is the relation between a false theory and its corresponding true theory. The explanandum, on the other hand, is the success of a false theory, which is, as I claimed earlier, a relation among a theory, observables and us.

Why is Stanford's suggestion of our interest? We want to know if we have good reason to believe that successful scientific theories are approximately true, and that their key terms refer. Scientific realists claim that we have such reason: Approximate truth and reference provide the best of explanation of the success of science. Scientific antirealists retort that we can explain the success of science without being forced into forming the realist belief. Arthur Fine, for instance, claims that a theory is successful because it is empirically adequate (1986) and instrumentally reliable (1991). He (1991: 82) goes further: The antirealist explanations are better than the realist explanation, contra scientific realists, because they take less epistemic risk than the realist one. Stanford's proposal is different from those of Fine. Stanford brings in the realist semantic property, truth, whereas Fine uses only the antirealist semantic properties, empirical adequacy and instrumental reliability. In any event, these proposals, Fine's and Stanford's alike, are intended to undercut the no-miracle argument, the best argument, for scientific realism.

Stanford's antirealist explanation is well-fortified against an empirical objection. Scientific realists might attempt to come up with counter-examples: successful theories in the history of science that are not sufficiently observationally similar to their corresponding true theories. These theories would block Stanford's inference from success to sufficient observational similarity. This is analogous to Laudan's strategy (1981) to block the realist inference from success to approximate truth. Laudan provides the infamous list of successful past theories, such as the caloric theory of heat and the ether theory. These theories, he claims, are not even approximately true because their key terms do not refer. Stanford's antirealist explanation, however, is not susceptible to the empirical objection in the way Putnam's realist explanation is for the following two reasons:

First, it is hard to find successful theories in the history of science that are not sufficiently observationally similar to their true theories. Can the successful past theories on Laudan's list serve as counter-examples against Stanford's antirealist explanation? It is clear that they turned out to be empirically inadequate, for they ran into anomalies. As Kuhn pointed out, the accumulation of anomalies is an importance force driving a scientific revolution. But that historical fact is compatible with the contention that they are sufficiently observationally similar to their true theories. After all, 'sufficient observational similarity' does not mean empirical adequacy. It means simply that the predictions of the successful theory are close to those of its true theory, i.e., the theory is close to being empirically adequate. 
The past theories on Laudan's list might indeed be far from being empirically adequate. But it is hard to prove them to be so.

Second, even if the past theories are obviously far from being empirically adequate, there is still a problem in using them to undercut Stanford's inference from success to observational similarity. They would undermine not only Stanford's inference but also the scientific realists' inference from success to approximate truth. After all, to say that a theory is approximately true (is likely to) involve the belief that it is sufficiently observationally similar to its true theory. If any scientific realists think otherwise, they need to answer this question: Is it plausible that the predictions of an approximately true theory are not close to those of its true theory? Moreover, if success cannot establish sufficient observational similarity, how can it establish approximate truth? In the absence of convincing answers to these questions, an empirical test is a bankrupt avenue for scientific realists, even if the counter-examples are abundant in the history of science. It looks like Stanford has built up an unassailable fortress against scientific realists.

How does Psillos (2001) tackle the fortress? Let me focus on two of his criticisms which I take to be central. The first criticism is that he doubts that "Stanford's story is genuinely explanatory" (2001: 348). His main premises for the objection are 1. that the approximate truth of the false theory explains its sufficient observational similarity to its corresponding true theory, and 2. that "the weight of the explanation of its success should be carried by the (approximate) truth" of the theory itself whose success is to be explained (2001: 350). The first premise is plausible. It makes sense that a false theory is sufficiently observationally similar to its corresponding true theory because it is approximately true. After all, an approximately true theory could be empirically adequate or approximately empirically adequate ${ }^{2}$; an (approximately) empirically adequate theory is necessarily sufficiently observationally similar to its corresponding true theory. The problem with Psillos's objection, however, is that although approximate truth explains sufficient observational similarity, it does not follow that sufficient observational similarity is not explanatory. A theory's explanatory power is independent of whether or not it is explained by another theory. Consider that from the fact that a chemical theory is explained by a physical theory, it does not follow that it is not explanatory. The chemical theory could still adequately explain a lot of phenomena regardless of whether it is explained by a more fundamental theory. I am not claiming here that observational similarity has an explanatory potency. Rather, my point is that Psillos's inference is incorrect. His incorrect inference is that observational similarity does not have explanatory potency because it can be explained by approximate truth.

The second premise is a bold claim that needs to be justified. Why is it that the weight of the explanation should be carried by the approximate truth? Suppose that scientific realists are right in saying that we have good reason to believe that successful scientific theories are approximately true. Even so, scientific antirealists would explain the success of the theories, not in terms of the approximate truth, but in terms of the sufficient observational similarity to their corresponding true theories. The motivation for giving such an explanation is that it is epistemically safer to appeal to the sufficient observational 
similarity than to the approximate truth. That is, the antirealist belief that successful theories are sufficiently observationally similar to their corresponding true theories is less likely to be false than the realist belief that they are approximately true. Fine (1991: 82), as we have seen earlier, would add that the less epistemic risk justifies the claim that their explanation is better than the realist explanation. ${ }^{3}$

Let me turn to Psillos's second objection that Stanford's story is reducible to absurdity. Psillos contends that observational similarity is not an adequate explanatory property for success on the following grounds:

Suppose--as we would intuitively--that Newton's theory explains the success of Kepler's and that Kepler's explains the successes of Copernicus's. Then, just because predictive similarity is not transitive we cannot infer that Newton's theory also explains the successes of the Copernican theory. In fact, Newton's theory does not even explain the success of Kepler's, on Stanford's account. For even if Kepler's theory is predictively similar to the true theory $\mathrm{T}$, and even if Newton's theory is predictively similar to T, it does not follow that Kepler's theory is predictively similar to Newton's. So, Newton's theory cannot even be a candidate for the explanation of the success of Kepler's theory. (Psillos, 2001: 354)

In other words, we cannot infer the observational similarity between Kepler's theory and Newton's theory from the observational similarity of Kepler's theory and Newton's theory to the true theory. The objection, however, is based on an uncharitable interpretation of Stanford's work. Stanford is not saying that a false theory is just observationally similar to its corresponding true theory. Rather, he is saying that the false theory is sufficiently observationally similar to the true theory. The following passage indicates how we should understand sufficient observational similarity:

...we do indeed explain the success of (revised) Ptolemaic system of epicycles by pointing out how closely its predictions approximate those of the true Copernican hypothesis. (2000: 273)

The predictions of the Ptolemaic hypothesis, Stanford states, approximate those of the Copernican hypothesis. It necessarily follows that the Ptolemaic hypothesis is approximately empirically adequate, given that the Copernican hypothesis is empirically adequate. So, on Stanford's account, the Ptolemaic hypothesis is observationally similar to the Copernican hypothesis to the degree that it is approximately empirically adequate. In other words, from the fact that a false theory is sufficiently observationally similar to its true theory, we can deductively infer that the false theory is approximately empirically adequate.

Stanford could develop his explanation of the success of Kepler's theory in terms of Newton's theory as follows: Kepler's theory is successful because it is sufficiently observationally similar to 
Newton's theory, which is approximately empirically adequate. Any theory whose predictions approximate those of an approximately empirically adequate successful theory is likely to be successful. So the inductive-statistical model of explanation in which an explanandum probably follows from an explanans is employed to explain the success of Kepler's theory in terms of the sufficient observational similarity between Kepler's theory and Newton's theory. Thus, there is no problem at all with the suggestion that Newton's theory is a candidate for the explanation of the success of Kepler's theory, contra Psillos.

Psillos might point out that the concept of sufficient observational similarity and the concept of approximate empirical adequacy are not precise. That is, it is not clear where we should draw the line between sufficient observational similarity and insufficient observational similarity, or between approximate empirical adequacy and complete empirical inadequacy. The criticism is fair enough. But so what? If these concepts are to be discarded because they are imprecise, the concept of approximate truth, which Psillos so often uses in the literature, is to be thrown away as well. So Psillos is not in a position to draw any formidable claim from the fact that the concepts are imprecise.

\section{Real Problems with Stanford's Proposal}

Now, I shall advance what I take to be real problems of Stanford's proposal. Let me begin with a minor problem. Stanford's proposal is silent about the success of true theories, and it is not clear how it could handle such cases. Why is, for example, the Copernican hypothesis successful? On Stanford's account, the story should go as follows: The Copernican hypothesis is successful because it is observationally similar to itself. What is wrong with such an explanation? Anything can be explained this way. A tennis ball is spherical because it is similar to itself with respect to shape. It is flat again because it is similar to itself with respect to shape. The sky is blue because it is similar to itself with respect to color. It is red again because it is similar to itself with respect to color. These explanations are all vacuous. The explanans is the very explanandum in disguise. Thus, Stanford's scheme of explanation yields vacuous explanations in the cases of the success of true theories.

How could Stanford reply to this criticism? He could say that, although his explanatory scheme cannot be applied to the success of true theories, it can be applied to the success of current theories, and that this is all that matters in the realism-antirealism debate. What reason would he adduce to justify the claim that successful current theories are false? He would point to the fact that successful past theories, such as the caloric theory of heat and the ether theory, are false. In fact, he (2001) advances a version of the pessimistic induction. Furthermore, Psillos (1996), one of the leading realists in the literature, has settled for the approximate truth of current scientific theories in the light of Laudan's historical objection (1981) to scientific realism. He claims that the scientific "claims of truthlikeness extend only to the theoretical constituents that essentially contribute to the successes of theories" (1996: S113). Since 
approximate truth is falsity, realists should not have a problem with Stanford's applying his explanatory scheme to the success of current theories.

Let me present what I take to be my best shot against Stanford's fortress. Suppose that a property, $\mathrm{P} 1$, is ontologically dependent upon another property, P2, i.e., P1 cannot exist without P2, but not vice versa. Then, I shall call P1 P2's dependent property, and P2 P1's underlying property. We should respect the ontological order between properties when we explain a property in terms of another property. A conceptual problem arises if we explain an underlying property in terms of a dependent property. It sounds odd, for instance, to assert that a tennis ball is spherical because it is similar to a baseball with respect to shape. The oddity stems from the fact that the tennis ball's being spherical is the underlying property of its being similar to the baseball with respect to the shape, and that the tennis ball's being similar to the baseball with respect to shape is the dependent property of its being spherical. The tennis ball could be spherical without being similar to the baseball. Just imagine a possible world where there are no baseballs. Even in such a world the tennis ball could be spherical. But the tennis ball cannot be similar to the baseball with respect to shape unless it is spherical in the first place. So we would rather say that the tennis ball is similar to the baseball with respect to shape because, among other things, it is spherical. In this example, the underlying property is an intrinsic property; the dependent property is a relational property.

But the ontological dependence between properties holds even between relational properties. For example, my property of being a son and my property of being similar to my brother with respect to the way we are related to our parents are both relational properties. But the former is the latter's underlying property; the latter is the former's dependent property. I can be a son of my parents even if I do not have a brother. But I cannot be similar to my parents with respect to the way I and my brother are related to them unless I am their son in the first place. My property of being a son of my parents is the ontological basis for my property of being similar to my brother with respect to the way we are related to our parents. So a conceptual problem arises when we explain the underlying property in terms of the dependent property. It sounds odd that I am a son of my parents because I am similar to my brother with respect to the way we are related to our parents. Such an explanation is like putting a cart in front of a horse. So rather we should say that I am similar to my brother with respect to the way we are related to our parents because, among other things, I am a son of my parents.

How is the truth of most observational consequences related to observational similarity? Both are relational properties. The former derives from a relation between a false theory and observables; the latter derives from a relation between the false theory and the true theory. The former, I claim, is the latter's underlying property; the latter is the former's dependent property. Most observational consequences of a false theory can be true even if it is not observationally similar to its true theory at all. Imagine a possible where there are no true theories. ${ }^{4}$ Even in such a world the observational consequences can be true. The point is that the observational consequences of a false theory are true or false independently of how the 
false theory is related to the true theory. But the false theory cannot be sufficiently observationally similar to the true theory unless most of its observational consequences are true in the first place. Therefore, (A) is good; (B) is bad.

(A) A false theory is sufficiently observationally similar to its true theory because most of its observational consequences are true.

(B) Most observational consequences of a false theory are true because it is sufficiently observationally similar to its true theory.

In other words, we should explain the observational similarity in terms of the truth of most observational consequences, not the other way around.

Now, let me raise an important question: How is success related to observational similarity? Recall that to say that a theory is successful is to say that at least some observational consequences are true, and that we have recognized them to be so. What is important for my purpose here is that the truth of some observational consequences is built into success. So a constituent of success is the partial ontological basis of observational similarity. It follows that it is also putting a cart in front of a horse to explain success in terms of observational similarity. Stanford's proposal is conceptually flawed.

I do not want to claim, though, that a false theory is observationally similar to its true theory because it is successful. There are two reasons for my refusal to explain the observational similarity in terms of the success. First, at least most, not some, observational consequences are required to be true in order for the false theory to be sufficiently observationally similar to the true theory. That is, the sufficient observational similarity does not arise from the truth of some observational consequences. Second, to say a false theory is sufficiently observationally similar to its true theory because it is successful gives the impression that success is required for a false theory to be observationally similar to its true theory when it is not. A false theory, which is sufficiently observationally similar to its true theory, need not be successful at all. That is not because observational consequences need not be true but because we need not recognize the observational consequences to be true. Suppose that a theory is empirically adequate, but that none of its observational consequences are ascertained to be true due to current technological difficulties. Such theory is not successful, even if it is sufficiently observationally similar to the true theory.

\section{Conclusion}

A conceptually sound explanation respects the ontological order between properties. We should explain a dependent property in terms of an underlying property, not the other way around. Stanford claims that a false theory is successful because it is sufficiently observationally similar to its corresponding true theory. 
The observational similarity, however, is the dependent property of the truth of the observational consequences; the truth of observational consequences is the underlying property of the observational similarity. So it is conceptually problematic to explain the truth of observational consequences in terms of the observational similarity. Given that the truth of some observational consequences is built into success, it is also conceptually flawed to explain the success of a false theory in terms of its observational similarity to the true theory. Thus, one of recent antirealist objections to the no-miracle argument is gone.

The core claim of this paper, however, goes against the contextual theory of explanation by van Fraassen (1980). The theory holds, roughly, that an explanation is an answer to a why-question, and that the appropriateness of the answer depends on context: interests and contrast-class. So it is appropriate, in some contexts, to explain the length of a flagpole in terms of the length of its shadow. Likewise, it should be appropriate, he would say, to explain an underlying property in terms of a dependent property in some contexts. This is an interesting criticism. It is, however, beyond the scope of this paper to criticize the contextual theory of explanation, and to develop the main thrust of this paper into a full-fledged theory of scientific explanation. I believe that it can be developed into a theory that is distinct from Salmon's causal theory of explanation (1978), although there would be some similarity between them. I, however, leave this task for a future occasion.

\section{End Note}

${ }^{1}$ Stanford uses 'predictive similarity' instead of 'observational similarity.' I believe that 'observational similarity' is better than 'predictive similarity' for what he has in mind. Consider that the theory of evolution is extremely successful. After all, it explains a wide variety of phenomena. But it does not predict very much. So to say that it is successful because it is predictively similar to its corresponding true theory is to miss the main reason why it is successuful.

${ }^{2}$ Kukla (1996) is the first to use 'approximate empirical adequacy' in the literature. But he does not define it. I would say that a theory is approximately empirically adequate when most of its observational consequences are true, or when each of its observational consequences is approximately true. Of course, it is hard to draw a line between approximate empirical adequacy and complete empirical inadequacy. Yet, it is a useful concept. Suppose that the pessimistic induction is strong, i.e., successful current theories will turn out to be false as successful past theories did. Then, successful current theories will turn out to be 
empirically inadequate too. Even so, constructive empiricists could say that they are approximately empirically adequate.

${ }^{3}$ Although Fine (1991: 82) would happily say so, I do not agree with him, for there are problems with the principle of economy. See Park (2001, Chapter 4) for the problems. In any case, Psillos does not consider this possible antirealist reply to him.

${ }^{4}$ It is possible that there could be a world with no true theories, given that anything but contradition can happen in a possible world. No contradiction is involved with the suggestion that there are no true theories.

\section{References}

Arabatzis, T. (2001), “Can a Historian of Science Be a Scientific Realist?," Philosophy of Science 68 (Proceedings) S531-S541.

Fine, A. (1986), "Unnatural Attitudes: Realist and Instrumentalist Attachments to Science.” Mind 95: 149179.

Fine, A. (1991), "Piecemeal Realism.” Philosophical Studies 61: 79-96.

Kitcher, P. (1993), The Advancement of Science. New York: Oxford University Press. 
Kukla, A. (1996), “Antirealist Explanations of the Success of Science.” Philosophy of Science 63 (Proceedings): S298-S305.

Laudan, L. (1981), “A Confutation of Convergent Realism.” Philosophy of Science 49: 19-49.

Park, S., (2001), Scientific Realism Vs. Scientific Antirealism. Ph.D. Dissertation, The University of Arizona.

Psillos, S., (1996), "Scientific Realism and the 'Pessimistic Induction." Philosophy of Science 63 (Proceedings): S306-S314.

(1999), Scientific Realism: How science tracks truth. New York: Routledge.

(2001), "Predictive Similarity and the Success of Science: A Reply to Stanford." Philosophy of Science 68: 346-355.

Putnam, Hilary (1975). Mathematics, Matter and Method (Philosophical Papers, vo. 1), Cambridge: Cambridge University Press.

Salmon, W. (1978), “Why Ask, “Why?”? An Inquiry Concerning Scientific Explanation,” Proceedings and Addresses of the American Philosophical Association, Vol. 51, No 6: 683-705.

Stanford, K. (2000), “An Antirealist Explanation of the Success of Science.” Philosophy of Science 67: 266-284.

(2001), "Fool's Errand, Devil's Bargain: What Kind of Underdetermination should We Take Seriously?" Philosophy of Science 68 (Proceedings): S1-S12.

van Fraassen, B. (1980), The Scientific Image. Oxford: Oxford University Press. 